\title{
Need of Physical and Chemical Restraints: Experiences at Inpatient Psychiatric Ward in a Tertiary Care Hospital in Karachi, Pakistan
}

\author{
Humera Saeed1, M. Shameel Khan1, Syeda Maheen Batool3, Asma Akbar Ladak3, Nadia Karim² and Rabeeka Aftab1
}

\begin{abstract}
In psychiatry, agitated / aggressive patients are often treated with de-escalation techniques. If this does not work, physical or chemical restrains are required; but in the event of resistance, seclusion is applied. We report the findings of baseline study of experiences of physical and chemical restraints in a tertiary care hospital in Karachi, where 104 files were evaluated retrospectively. The mean age of patients was $32.5 \pm 14.3$ years with $54.8 \%$ men, while the average length of stay was $11.5 \pm 9.3$ days. Agitation, violent behaviour, and aggression were the most common indications for restraints. In total, $94.5 \%$ of patients had both physical and chemical restraints with the latter being used as the first choice in 70 patients; whereas, $67.1 \%$ of patients' families were not informed before application of restraints. The seclusion need assessment was conducted in $4.1 \%$ of patients.
\end{abstract}

Key Words: Restraint, Physical, Chemical, Compliance, Seclusion, Psychiatry.

Aggressive behaviours resulting from mental disorders are due to complex interactions between the inner world of a mentally ill person and his environment. ${ }^{1}$ Agitation is an excessive motor / verbal activity; but aggression is not fundamentally linked to agitation, which makes this relationship ambiguous. ${ }^{2}$ Extreme agitation / potentially violent behaviour can be dealt with de-escalation techniques, which along with environmental modifications are physicians' first choice. ${ }^{3}$

Chemical restraint / rapid tranquilization (RT) should be carefully administered to enable rapid and short-term behavioural control, which puts people at risk of harm to themselves or others, after failure of initial therapeutic interventions. ${ }^{4}$ The goal is to calm the person instead of treating the underlying psychiatric condition. 5

Physical restraint is any manual method of use of physical/ mechanical devices / material / equipment attached to a body in order to restrict movements/ freedom.

Seclusion is the sole confinement of the person at any hour of day or night in a room in which doors and windows are locked. It is the deliberate isolation of patient. Under these circumstances, the person is not able to reverse (Under s. 82 (1) of the Mental Health Act, UK).

1 Department of Psychiatry / Neurology and Neurosurgery ${ }^{2}$,

The Aga Khan University Hospital, Karachi, Pakistan

${ }_{3}$ Medical Student, The Aga Khan University Hospital, Karachi, Pakistan

Correspondence: Dr. Humera Saeed, Department of Psychiatry, The Aga Khan University Hospital, Stadium Road, Karachi, Pakistan

E-mail: humera.saeed@aku.edu

Received: August 20, 2018; Accepted: December 14, 2018
Physical restraint and isolation aim to "limit the movement and / or freedom of an individual to act independently". This can involve physical and psychological risks, including feelings of terror, shame, and helplessness, when the principles of autonomy, nonmaleficence and dignity of the patient are questioned.

In a prospective study of 289 psychiatric patients, Zun et al. reported a $7 \%$ complication rate in patients put under restraint. 6

In a developing country like Pakistan, where psychiatric inpatient care is mostly unregulated and unsupervised, adequate policies and legislations are needed as patients are at risk for abuse.

This was a descriptive study of retrospective design, at Aga Khan University Hospital (AKUH), Karachi. The duration of this study was from February 2015 to January 2016. The sample size comprised of 104 patients out of 477 patients admitted to psychiatric ward who received any form of restraint for the above duration. Case files were reviewed to obtain the relevant clinical information about practices of physical and chemical restraints and to find areas of improvement.

This study was conducted following ethical approval from AKUH, Karachi. Patients' confidentiality was maintained. Data were available only to the principal investigator and relevant team members.

Of the total 104 cases, $70.2 \%$ were primary and $29.8 \%$ repeated admissions. Final results were derived from 73 primary cases, of which $54.8 \%$ were males and $45.2 \%$ females. For majority of patients, hospital stay ranged from 1-15 days. Only $3(4.1 \%)$ patients were admitted for more than 30 days. Common admission indicators 
Table I: Characteristics of the study population.

\begin{tabular}{lc}
\hline Patients' characteristicis & $\begin{array}{c}\text { Mean } \pm \text { Standard deviation } \\
(\mathrm{n}=73)\end{array}$ \\
\hline Age (years) & $32.5 \pm 14.3$ \\
No. of admissions & $1.5 \pm 0.9$ \\
Length of stay (days) & $11.5 \pm 9.3$ \\
No. of restraints & $4.2 \pm 10.5$ \\
\hline
\end{tabular}

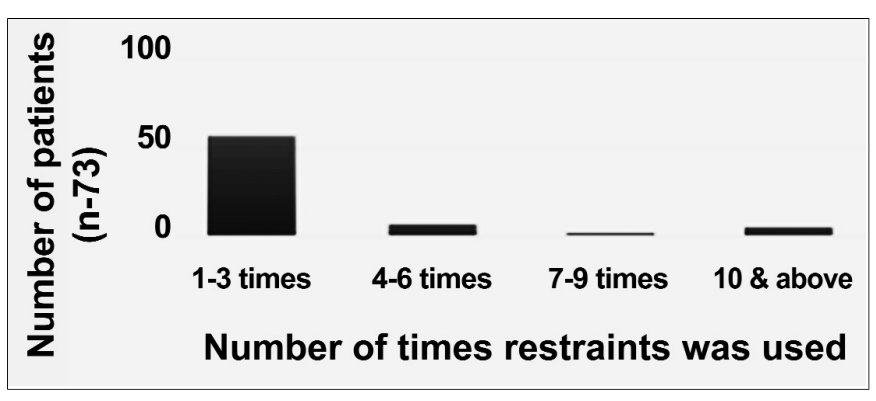

Figure 1: Number of times restraint was used per patient.

included bipolar disorder $33(45.2 \%)$, psychosis 20 (27.4\%), mood disorder $8(11.0 \%)$, substance dependence $8(11.0 \%)$, anxiety disorder $2(2.7 \%)$, and intellectual disability 2 (2.7\%).

Violent behaviour and aggression were the common indications for using restraint followed by psychotic episodes. A total of 69 patients received both physical and chemical restraint accounting for $94.5 \%$ of all cases. Chemical restraint alone was received by three patients only and one patient was subject to physical restraint only (Figure 1).

First restraint used in 70 patients was chemical, and 3 received physical type. Those subjected to restraint between 1 to 3 times were $79.5 \%$.

Majority of the patients were admitted for the first time. For a few inpatients, admission extended to more than a month; otherwise for most, it was between 1 and 15 days. Most common diagnosis was bipolar disorder followed by psychosis.

This study confirms the findings of other studies that violence and aggression are the most common indicator for use of restraint and majority of the patients received both chemical and physical restraints. Chemical restraint was used as first choice in nearly all.

An important issue, which is highlighted here, is that restraint is often necessary to ensure safety of the patient. Hence, there is a need for general hospitals in Pakistan to have robust policies, which will not only provide safe treatment but will also protect patients' rights.

Some of the domains which may require important consideration in a policy may include:

Chemical restraint/Rapid tranquillization (RT) should be used only for calming a patient following failure of verbal de-escalation and environmental modification. Throughout the process, a set of standard operating procedures (SOPs) need to be followed. These include maintaining the patients' privacy and dignity, informing the family, calling security when required, and under-standing of the line of authority, i.e. who will order for RT or physical restraint.

Considerable cautions must be exercised before applying restraint to any patient with any medical illness such as seizures. Safety measures must be taken before restraining the patient, which include removing sharp and harmful objects, side rails should be placed and patient must be wearing loose clothing.

There should be a written order; and close observation should be followed throughout, including hourly monitoring of circulation status.

In addition to RT, physical restraints and seclusions are classified as restrictive practices. Restrictive practices aim to 'restrict an individual's movement, liberty, and/or freedom to act independently without coercion or consequence'. However, physical restraint and seclusion may pose substantial physical and psychological risk including inducing feelings of terror and powerlessness.

In the absence of operational mental health act, general hospitals in Pakistan need to develop guidelines and policies, so that patients' rights are not violated.

In Pakistan, where there is little regulation on psychiatric practice, the potential of abuse of disturbed psychiatric patients by inappropriate and excessive use of both physical and chemical restrains may become an issue. Robust policies and effective legislations will secure patients' autonomy and ensure good and safe psychiatric practice.

There is a need for audits to measure good practice; and also to develop effective training programme to spread awareness among medical staff. Since the use of restraints may be necessary in psychiatric setting, a good surveillance system is also required in order to prevent its unnecessary use.

The limitations of the present study are that the sample size was not large and may not be the truly representative of all mentally ill subjects in Pakistan. However, the main emphasis of the present study was focused on introduction of proper surveillance system for restraints.

\section{REFERENCES}

1. McDermott BE, Holoyda BJ. Assessment of aggression in inpatient settings. CNS Spectrums 2014; 19:425-31.

2. de Almeida CG, Del Grossi Moura M, Barberato- Filho S, de Sa Del Fiol F, Motta RHL, de Cássia Bergamaschi C. Rapid tranquilization for psychiatric patients with psychomotor agitation: What is known about it? Psychiatr Q 2017; 88:885-95.

3. Garriga M, Pacchiarotti I, Kasper S, Zeller SL, Allen MH, 
Vazquez G, et al. Assessment and management of agitation in psychiatry: Expert consensus. World J Biol Psychiatry 2016; 17:86-128.

4. De Fruyt J, Demyttenaere K. Rapid tranquilization: New approaches in the emergency treatment of behavioral disturbances. Eur Psychiatry 2004; 19:243-9.
5. Hankin CS, Bronstone A, Koran LM. Agitation in the inpatient psychiatric setting: A review of clinical presentation, burden, and treatment. J Psychiatr Pract 2011; 17:170-85.

6. Zun LS. A prospective study of the complication rate of use of patient restraint in the emergency department. J Emerg Med 2003; 24:119-24.

....畺.... 